\title{
Lessons of the month 2: McKittrick-Wheelock syndrome: Case report of a patient declining resection of a large villous adenoma
}

\author{
Authors: Jagan Murugachandran ${ }^{A}$ and David Gannon ${ }^{B}$
}

\begin{abstract}
McKittrick-Wheelock syndrome (MWS) is a rare cause of acute kidney injury and electrolyte depletion. It is driven by a secretory tumour or polyp of the distal large colon. Although rare, it is a diagnosis that must be considered in patients presenting with the above conditions. Treatment is with surgical resection of the offending growth. We present the case of a 70-year-old woman who presented multiple times with electrolyte depletion and acute kidney injury. The diagnosis of MWS was established and treatment options discussed. Surgical intervention was declined, and management consisted of patient education, the careful replacement of fluid and electrolytes and close monitoring of electrolytes and kidney function. After a short period as an inpatient, the patient went on to be discharged and, with regular follow-up, has managed to continue a normal life.
\end{abstract}

KEYWORDS: McKittrick, Wheelock, villous adenoma, electrolyte depletion

DOI: 10.7861/clinmed.2019-0356

\section{Background}

McKittrick-Wheelock syndrome (MWS) is a rare cause of acute kidney injury (AKI) with electrolyte depletion (ED). First described in 1954 by McKittrick and Wheelock, the syndrome is defined by the constellation of ED, AKI and secretory diarrhoea driven by a large neoplastic lesion of rectum or rectosigmoid colon, typically a villous adenoma. Since its initial description, there have been multiple case reports and case series describing the condition. ${ }^{1-4}$ However, it remains a rare life-threatening entity that is poorly recognised and is associated with significant morbidity if not treated. ${ }^{4}$ Definitive treatment is resection of the offending lesion, this is often curative. Unfortunately, due to the time between presentation and diagnosis, treatment is often delayed.

Authors: ${ }^{\text {A }}$ medical registrar, Colchester General Hospital, Colchester, UK; ${ }^{\mathrm{B}}$ consultant acute physician, Colchester General Hospital, Colchester, UK

\section{Case presentation}

We report the case of a 70-year-old woman with background of hypertension, stage 3 chronic kidney disease and hypothyroidism, who presented to the emergency department of her local hospital with multiple episodes of AKI and ED.

In the weeks leading up to her first admission, the patient noticed significant lethargy, anorexia and weight loss. The week prior to presentation, her general practitioner diagnosed a lower respiratory tract infection and she was started on antibiotics for this.

On the day of her sentinel presentation, she was brought to the emergency department after being found collapsed and acutely confused. On examination, the patient had a normal respiratory and abdominal exam but was hypotensive with clinical signs of dehydration. There was no history of head injury and, although stuporous, the patient was easily rousable.

Her baseline investigations included electrocardiography demonstrating normal sinus rhythm with a rate of 60 beats $/ \mathrm{min}$, an arterial blood gas demonstrating a mixed alkalosis with $\mathrm{pH}$ of 7.581, arterial partial pressure of carbon dioxide of $3.96 \mathrm{KPa}$, arterial partial pressure of oxygen of $9.35 \mathrm{KPa}$, sodium of 104 $\mathrm{mmol} / \mathrm{L}$, potassium of $2.8 \mathrm{mmol} / \mathrm{L}$, base excess of $6.2 \mathrm{mmol} / \mathrm{L}$, and chloride below measurable range $(<70 \mathrm{mmol} / \mathrm{L})$. A formal lab workup identified a sodium of $103 \mathrm{mmol} / \mathrm{L}$, potassium of 3.8 $\mathrm{mmol} / \mathrm{L}$ and stage 2 AKI. The patient was transferred to critical care for monitoring and replacement of fluids and electrolytes. Over the following several days the patient improved. After 5 days, she was discharged home with a working diagnosis of dehydration and AKI secondary to poor oral intake, a lower respiratory tract infection and ongoing use of candesartan and omeprazole.

The patient re-presented 2 weeks post discharge with a fall, malaise and decreased mobility. Further questioning at this time elicited an acute-on-chronic history of a diarrhoeal illness dating back several years. During this time, she had an intermittent history of loose stool, with approximately 2-3 bowel motions per day. The stool was described as watery with no associated blood or mucous. Two years prior to presentation the patient underwent computed tomography (CT) of the colon to investigate her diarrhoea. This had identified abnormalities in the sigmoid warranting further investigation. However, this was not pursued due to patient wishes. Despite the chronic loose stool, the patient remained well in herself and had very little interaction with health services over the following 2 years. 

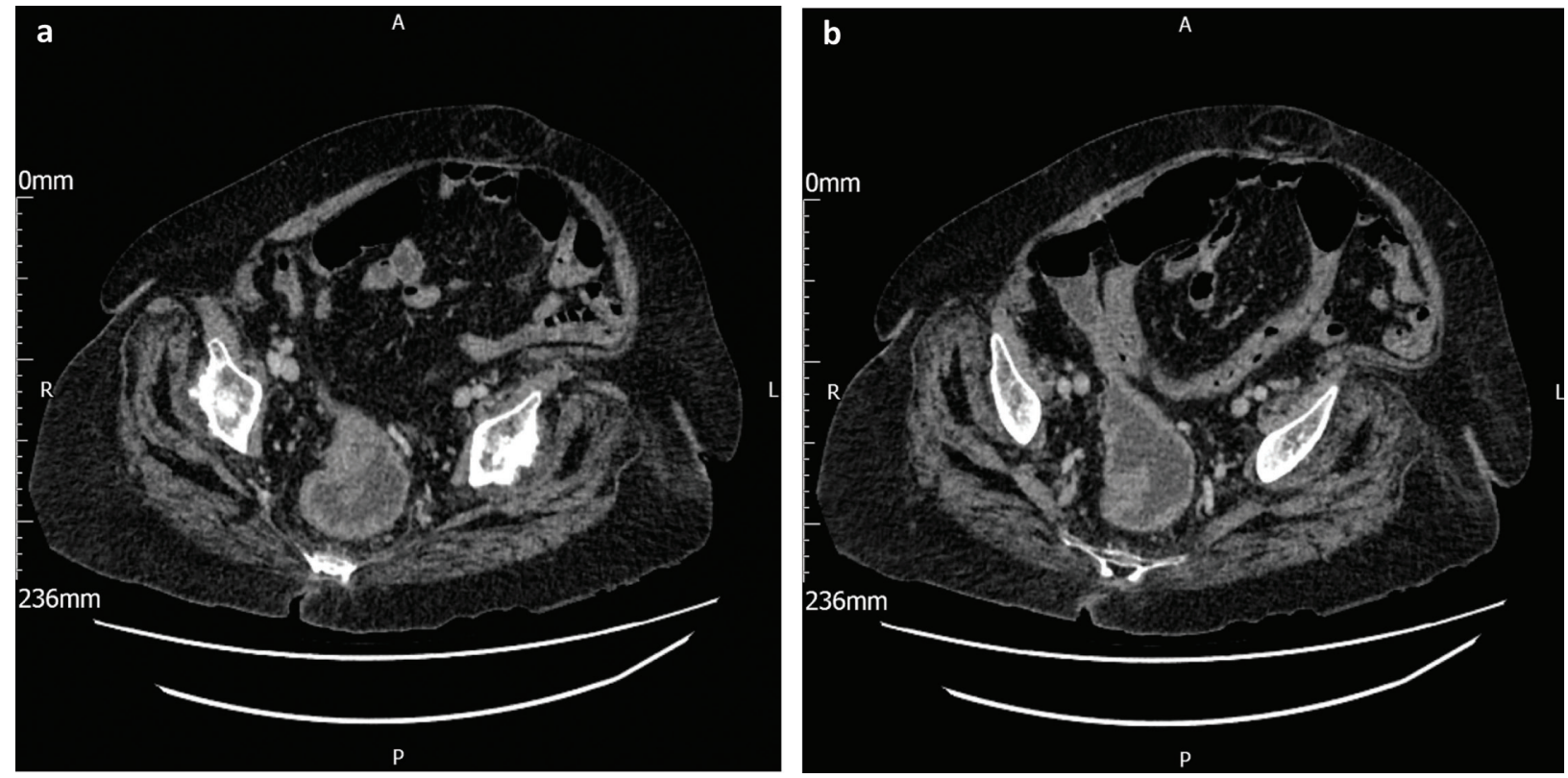

Fig 1. Computed tomography demonstrating a fluid filled rectum with asymmetric thickening of the sigmoid wall.

During the second admission the patient underwent further CT (Fig 1) demonstrating a fluid filled rectum with asymmetric thickening of the sigmoid wall. The patient underwent flexible sigmoidoscopy which identified a large villous adenoma at the border of the rectosigmoid junction descending into the rectum.

In view of the findings, a diagnosis of MWS was made. Surgical resection of the villous adenoma was offered to the patient, however further investigation and intervention was declined. Over the course of the next 23 days, there was improvement in renal function back to baseline as well as normalisation of her serum electrolytes.

During her stay, the patient was reviewed by dietetics and offered advice regarding hydration and electrolyte replacement strategies in the community; patient and family education was also given to ensure recognition of symptomatic deterioration.

Unfortunately, in the following month, the patient presented with cellulitis affecting her left leg and once again had developed
ED and AKI. This responded quickly to treatment of her cellulitis, with concurrent intravenous replacement of fluids and electrolytes.

\section{Discussion}

MWS is classically attributed to a large secretory villous adenoma of the sigmoid or rectum. There are, however, cases due to adenocarcinoma and neuroendocrine tumours of the rectum., ${ }^{1,2}$

Treatment of MWS involves initial resuscitation and replacement of electrolytes and fluids. Definitive treatment is surgical resection of the bowel lesion. Non-steroidal anti-inflammatory drugs, such as indomethacin, are thought to provide some benefit by suppressing prostaglandin-E2 activity. ${ }^{5}$ Somatostatin analogues have been shown to reduce diarrhoeal volume in villous adenoma disease and may have a role in preventing ED.

Unfortunately, the incidence of malignant transformation remains high in villous adenomas as does the risk of morbidity due to pre-renal AKI and electrolyte depletion. ${ }^{4,6}$ On performing
Fig 2. Variations in serum electrolytes and creatinine over the course of three acute presentations to hospital. Each coloured background corresponds to each presentation.

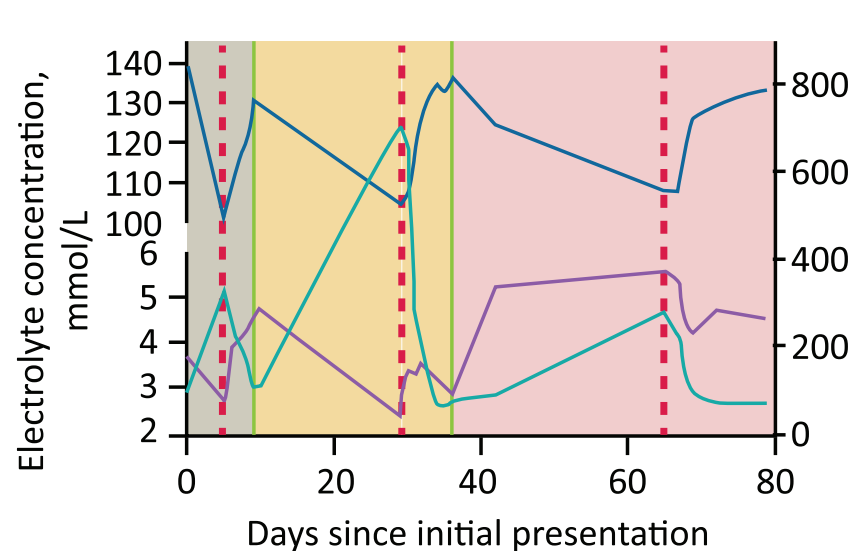

Days since initial presentation 
a PubMed search, there are two case reports of patients declining surgical intervention; one patient with a metastatic neuroendocrine tumour received radiotherapy and chemotherapy with good response, the other was complicated by bacterial endocarditis with associated mortality., ${ }^{1,2}$

This case highlights the complexities arising from MWS. Patient and family awareness of the condition, its symptomatology, home treatment strategies and indications for admission can reduce morbidity and can promote patient stability. Stability is ultimately determined by the balance between fluid and electrolyte intake as well the secretion load of the tumour. It is the changes in this balance that can lead to decompensations necessitating admission for intravenous fluid and electrolyte replacement.

Peaks and troughs relating to electrolytes and kidney function (Fig 2) correlate to deterioration in the patient and hospital presentations. There are multiple causes for decompensation, these include behavioural and physiological responses to injury; this was the case in our patient, where decompensation was driven by reduced oral intake due to a lower respiratory tract infection and an episode of cellulitis.

\section{Conclusion}

Clinicians must be aware of MWS, awareness of the condition and its pathophysiology allow the clinician to anticipate patient deteriorations. Finally, although not curative, close monitoring and careful management of electrolytes can lead to a satisfactory quality of life for those with MWS.

\section{References}

1 Dahya V, Dahya Z, Cua Y. McKittrick-Wheelock syndrome complicated by Enterococcusfaecalis endocarditis: a unique combination. Int J Colorectal Dis 2015;30:285-6.

2 Nakhla SG, Murakami TT, Sundararajan S. Poorly differentiated neuroendocrine tumor of the rectum coexistent with giant rectal villous adenoma presenting as McKittrick-Wheelock syndrome. Case Rep Oncol Med 2015;2015:242760.

3 Dagan A, Reissman P. Giant secretory villous adenoma of the rectum and sigmoid presenting as McKittrick-Wheelock syndrome. Int J Colorectal Dis 2010;25:909-10.

4 Popescu A, Orban-Schiopu A-M, Becheanu G, Diculescu M. McKittrick-Wheelock syndrome - a rare cause of acute renal failure. Romanian J Gastroenterology 2005;14:63-6.

5 Steven K, Lange P, Bukhave K, Rask-Madsen J. Prostaglandin E2-mediated secretory diarrhea in villous adenoma of rectum: Effect of treatment with indomethacin. Gastroenterology 1981:80:1562-6.

6 Amersi F, Agustin M, Ko CY. Colorectal cancer: epidemiology, risk factors, and health services. Clin Colon Rect Surg 2005;18:133-40.

Address for correspondence: Dr Jagan Murugachandran, Colchester General Hospital, Turner Road, Colchester CO4 5JL, UK.

Email: jaganmcn@doctors.org.uk

\section{Royal College} of Physicians

\section{Looking for jobs in medicine?} Find your next hospital job

Find out more and subscribe to the monthly Medicine Jobs bulletin at www.rcplondon.ac.uk/jobsboard 